\title{
Effect of Extenders and Insemination Protocol on the Fertilizing Capacity of Cryopreserved Arabian Horse Semen
}

\author{
M.M. Waheed ${ }^{1,2 *}$, K.O. Al-Khaldi ${ }^{3}$, and N. Pratap ${ }^{4}$ \\ ${ }^{1}$ Department of Clinical Studies, College of Veterinary Medicine, King Faisal University, Al-Ahsa 31982, P.O. 400, Al-Hufof, Kingdom of Saudi Arabia \\ ${ }^{2}$ Department of Theriogenology, Faculty of Veterinary Medicine, Cairo University, P.O. 12515, Giza, Egypt \\ ${ }^{3}$ Royal Oman Police, Mounted Division, Veterinary Section, Muscat, Oman \\ ${ }^{4}$ Division of Reproduction, Laboratories and Animal Research Centre, Directorate General of Veterinary Services, Royal Court Affairs, P.O. Box 64, P.C. \\ 111, Muscat, Oman
}

Received: September 12, 2016; Accepted: September 21, 2016; Published: September 24, 2016

*Corresponding author: Magdi Waheed, College of Veterinary Medicine, King Faisal University, Al-Ahsa 31982, P.O. 400, Al-Hufof, Kingdom of Saudi Arabia, Tel: +966-135-816600; Fax: +966-135-896617; E-mail address: mmwaheed@kfu.edu.sa

\begin{abstract}
Forty-eight ejaculates were obtained from four Arabian stallions to study the impact of three extenders (INRA Freeze, TRIS-egg yolk and E-Z Mixin) on the fertilizing capacity of cryopreserved stallions semen. Ejaculates were cryopreserved in 0.5 -ml plastic straws with a dilution rate of $1: 1$ and 1:2. Frozen straws were thawed at either $37^{\circ} \mathrm{C}$ for $30 \mathrm{sec}$ or $70^{\circ} \mathrm{C}$ for $7 \mathrm{sec}$ and used for AI of eighty Arabian mares via different protocols. Results revealed that percentages of motility; live sperm; and abnormalities were significantly $(P<$ 0.01 ) better in the E-Z Mixin at $5^{\circ} \mathrm{C}$ with dilution rates 1:1 and 1:2. Motility characteristics such as path velocity (VAP, $\mu \mathrm{m} / \mathrm{s})$, straightline velocity (VSL, $\mu \mathrm{m} / \mathrm{s})$, Point-to-Point Velocity $(\mathrm{VCL}, \mu \mathrm{m} / \mathrm{s})$ and lateral head displacement $(\mathrm{ALH}, \mu \mathrm{m})$ were significantly $(P<0.01)$ the best by using E-Z Mixin at rates of dilution 1:1 and 1:2. Conception rate was significantly $(P<0.01)$ higher by using INRA Freeze that had thawed at $37^{\circ} \mathrm{C}$ at dilution rate $1: 1(50 \%)$ than at dilution rate $1: 2(20 \%)$. The use of two inseminating doses gave significantly $(P<$ 0.01 ) better results $(50 \%)$ than did the use of one inseminating dose (28.57\%). The fertilizing capacity of cryopreserved Arabian horse semen was best by using INRA Freeze at a dilution rate $1: 1$ and two insemination doses.
\end{abstract}

Keywords: Arabian horse; Semen; Extenders; Cryopreservation; Fertilizing capacity

\section{Introduction}

Artificial insemination is considered one of the modern ways of achieving genetic improvement and the improved production potential of horses [1,2]. Levels of success in breeding with frozen semen depend on interaction between the fertility of the stallion, the fertility of the mare and managerial practices [3]. The first pregnancy in a mare inseminated with frozen semen was reported in 1957, but frozen horse semen became more widely used only in the late 1980s [4]. Indeed, pregnancy itself is the best tool for assessing stallion fertility [5]. A large population of stallions still has poor semen quality and low levels of fertility after freezing thawing; as a result, a number of modifications have been proposed for the freezing process [6]. It has been estimated that only $30-40 \%$ of stallions produce semen suitable for cryopreservation [7]. Moreover, consistent variation of sperm freezability has been observed from breed to breed [8,9]. At present, there is no standard protocol for stallion semen cryopreservation able to achieve consistent and acceptable post thaw sperm fertilizing capacity with every ejaculate [10]. That lack of standard can be attributed to many factors, including damage to the sperm membrane that may occur during the process of freezing or thawing [11]. In addition, the cryopreservation extender has an important influence on post thaw sperm quality [11].

Many diluents have been used in different animal species, with good results in post thaw sperm motility, but they have not been used with the semen of Arabian horses. Several diluents have been used in stallion semen, with good results such as Kenney's diluent, cream-gel, skim milk diluent, glycine egg yolk, sugar-based extender and INRA 96 extender [12,13]. The use of Computer- Assisted Semen Analysis (CASA), which facilitates objective measurement of several parameters of sperm motility, offers a more reliable and repeatable means of assessing sperm motility than does examination by eye [14]. The CASA system is therefore able to determine a series of variables, including number of moving spermatozoa, Curvilinear Velocity (VCL), Linear Velocity (VSL), Linear Coefficient (LIN), straightness coefficient (STR) and frequency of head displacement (BCF) [15].

Success with frozen semen requires attention to detail and a basic understanding of the techniques involved in the proper handling, thawing, and insemination of frozen semen [7]. Frozen stallion semen is now used more extensively by minimizing freeze thaw damage to spermatozoa, and indeed, more stallions might be used and pregnancy rates might be increased. Many steps constitute interactive participation in the success of the cryopreservation process, including semen collection, dilution, cooling, freezing, and thawing [12]. 
Common practice aims to inseminate the mare with frozen semen 6-12 h pre ovulation and $6 \mathrm{~h}$ post-ovulation. On one hand, insemination post ovulation requires examination of the mare at least every $6 \mathrm{~h}$, with the artificial insemination performed as soon as ovulation has been detected; this can be a very labor-intensive system [16-18]. On the other hand, insemination pre ovulation may result in wastage of expensive semen if the mare fails to ovulate within the expected time frame [19].

The present study was designed to evaluate the impact of diluents on semen processing, on postthaw progressive sperm motility, and on the fertilizing capacity of cryopreserved Arabian horse semen.

\section{Materials and Methods}

\section{Animals}

A total of four healthy Arab stallions, aged 6 to 12 years, were involved in this study. The stallions belong to the Mounted Division of the Royal Oman Police, in Muscat, Oman (23- 37'-N, $\left.58^{\circ}-35^{\prime}-E\right)$ and have been used as sires in the stables' regular breeding program. Each stallion was housed in a separate pen under standard conditions of feeding and general management. The stallions were fed three times daily, and water was freely available. All four animals were exercised daily for at least $1 \mathrm{~h}$ Eighty Arabian mares ranging from 6 to 15 years old with good histories of fertility were used for the artificial insemination.

\section{Semen collection and evaluation}

Forty-eight ejaculates were collected from the stallions using Hanoverian Equine Artificial Vagina Kit (Minitube of America, Verona, WI, USA) during the breeding season (October to April) while the stallions mounted mares in estrus. Immediately after collection, sperm-rich ejaculate fraction (fractionated by the kit filters) from each stallion was transferred to the laboratory, placed in a water bath at $30^{\circ} \mathrm{C}$ and assayed by computer-assisted semen analysis equipment (Hamilton Thorne Research, Beverly, MA, USA) [20]. Sperm motility characteristics, sperm concentration, percentages of live sperm and sperm abnormalities were determined as described earlier [21]. In addition, the following characteristics of sperm motility were estimated: velocity of the sperm path (VAP, $\mu \mathrm{m} / \mathrm{s}$ ); average velocity of a straight line of the sperm track (VSL, $\mu \mathrm{m} / \mathrm{s}$ ); average velocity of the actual point-topoint track followed by the sperm cell (VCL, $\mu \mathrm{m} / \mathrm{s}$ ); amplitude of lateral head displacement $(\mathrm{ALH}, \mu \mathrm{m})$; beat-cross frequency $(\mathrm{BCF}$, $\mathrm{Hz}$ ); straightness (STR, \%, or average value of the ratio VSL/VAP), which measured the sperm path's departure from a straight line; and linearity (LIN, \%, or average value of the ratio VSL/ VCL) which measured the sperm path's departure from a straight line [22]. Those sperm parameters were determined after semen dilution and cooling to $5^{\circ} \mathrm{C}$, after having thawed at $37^{\circ} \mathrm{C}$ for 30 sec and having thawed at $75^{\circ} \mathrm{C}$ for $7 \mathrm{sec}$.

\section{Semen dilution and freezing}

All chemical compounds used in this study were purchased from Merck (Merck, Darmstadt, Germany) unless otherwise stated.
The extenders used were (1) INRA Freeze (IMV, Paris); (2) TRIS -egg yolk $(2.4 \mathrm{~g}$ TRIS $+1.25 \mathrm{~g}$ citric acid $+0.45 \mathrm{~g}$ glucose $+22 \mathrm{~mL}$ egg yolk $+4 \mathrm{~mL}$ glycerol and deionized water to $100 \mathrm{~mL}+$ antibiotics "penicillin G sodium $50000 \mathrm{IU}$ and dihydrostreptomycin $50 \mathrm{mg}$ "); and (3) E-Z Mixin BF (Colorado, USA; 340388): $125 \mathrm{~mL}$ E-Z Mixin + $4 \mathrm{~mL}$ glycerol and antibiotics. Each sperm-rich ejaculate fraction was divided into two aliquots. One aliquot was diluted at a rate of $1: 1$, and the other at a rate of $1: 2$ at $30^{\circ} \mathrm{C}$ using the aforementioned extenders. Briefly, sperm-rich fractions were diluted and centrifuged at $1500 \mathrm{~g}$ for $10 \mathrm{~min}$. Then the supernatant was decanted, and sperm pellets reconstituted with the same portion of the extenders previously added. The diluted semen was cooled to $5^{\circ} \mathrm{C}$ over $75 \mathrm{~min}$. The cooling was around $0.3^{\circ} \mathrm{C} / \mathrm{min}$. At $5^{\circ} \mathrm{C}$, the diluted semen was packed in $0.5-\mathrm{ml}$ French straws (the sperm cell concentration ranged from 25 to $40 \mathrm{sperm} / \mathrm{straw}$ ). The straws were frozen in a programmable freezer (SY-LAB- ICE CUBE 1810 computer freezer, Minitube, Burladingen, Germany) to minus $140^{\circ} \mathrm{C}$ within $7 \mathrm{~min}\left(20.7^{\circ} \mathrm{C} / \mathrm{min}\right)$ and stored in liquid nitrogen at minus $196^{\circ} \mathrm{C}$. Frozen straws were thawed at either $37^{\circ} \mathrm{C}$ for 30 sec or $75^{\circ} \mathrm{C}$ for $7 \mathrm{sec}$ and incubated at $30^{\circ} \mathrm{C}$ for $3 \mathrm{~h}$. The thawed samples were compared between extenders, rates of dilution, thawing temperatures in terms of sperm motility, concentration, percentage of live sperm, percentage of sperm abnormalities and viability indexes. The viability indexes [23] were computed by the following equation:

$$
\mathrm{VI}=\sum[\mathrm{Mx}(\mathrm{T}-\mathrm{R} / 2)]
$$

Where, VI is the viability index, $\sum$ is a sign for the sum total, $\mathrm{M}$ is the percentage of sperm motility, $\mathrm{T}$ is the time of the next determination of motility, and $\mathrm{R}$ is the time of the previous determination of motility. The best extender with best thawing temperature and viability index was used for artificial insemination of Arabian mares, which was INRA Freeze, with a thawing temperature of $37^{\circ} \mathrm{C}$ for $30 \mathrm{sec}$.

\section{Assessment of cryopreserved arabian horse semen by artificial insemination}

Eighty Arabian mares aged 6 to 15 years with good histories of fertility, were used to test the fertilizing capacity of cryopreserved Arabian horse semen preserved in INRA Freeze. Mares were teased by using sexually desirous stallions that would detect mares in estrus. Estrous mares were examined daily via ultrasonography by direct manual manipulation of a convex multifrequency transducer (Aloka Prosound SSD 3500SX, ALOKA Co., Ltd., Tokyo) throughout the entire estrous period and twice daily when the mares were close to ovulation during the last 2 days of estrus and every $6 \mathrm{~h}$ around ovulation.

The mares were divided into two groups: one group was inseminated with frozen-thawed semen diluted in INRA Freeze at a rate of $1: 1$ and the other, at a rate of 1:2; all straws were thawed at $37^{\circ} \mathrm{C}$ for $30 \mathrm{sec}$. Every attempt was made to breed the mares within $6 \mathrm{~h}$ before ovulation and for some of them, to breed by two doses: one before and one $24 \mathrm{~h}$ after ovulation. Mares that had good estrous signs with marked uterine edema and a dominant follicle $>35 \mathrm{~mm}$ but that did not ovulate were 
given $2500 \mathrm{IU}$ I/V of human chorionic gonadotrophin (hCG; Chorulon, Intervet UK Limited, Milton Keynes, United Kingdom) and bred at $24 \mathrm{~h}$ and $40 \mathrm{~h}$ after hCG administration. At the time of insemination, a sterile, flexible pipette was advanced vaginally past the cervix and semen deposited at the body of the uterus. Volumes of $0.5-5 \mathrm{ml}$ of thawed semen with a minimum number of 300 million progressively motile and morphologically normal sperm were inseminated using air to flush the pipette [24]. All inseminated mares were examined for pregnancy at day 18 via ultrasonography and the conception rates were recorded.

\section{Statistical analysis}

Data of stallions' semen parameters and characteristics of sperm motility (24 ejaculates) in the used three extenders are presented as mean \pm standard error of the mean. The repeated measures of those parameters were compared by analysis of variance and chi-squared test (X2) Using SPSS statistical software program (2013), version 22.0 [25].

\section{Results}

The Arabian horses' fresh semen characteristics are shown in Table 1 as mean \pm SEM. The ejaculate volume, percentage of progressive motility, sperm cell concentration and percentage of sperm cell abnormalities were $60.00 \pm 3.34 \mathrm{ml}, 54.79 \pm 2.32 \%$, $178.63 \pm 19.47 \times 106 / \mathrm{ml}$ and $23.46 \pm 1.46 \%$, respectively. There is a highly significant $(P<0.01)$ difference between semen parameters in the different extenders after cooling of stallions' semen to $5^{\circ} \mathrm{C}$ (Table 2). The percentages of progressive motility, live sperm and sperm cell abnormalities were significantly better $(P<0.01)$ in the INRA 96 extender at rates of dilution of 1:1 $(39.54 \pm 2.50,63.68 \pm 1.54$ and $18.42 \pm 2.26$, respectively) and $1: 2(44.13 \pm 3.07,67.22 \pm 1.36$, and $27.33 \pm 1.8$, respectively) than in the other two extenders (TRIS -egg yolk and E-Z Mixin; Table 2).

The characteristics of the stallions' sperm motility at $5^{\circ} \mathrm{C}$ such as VAP $(\mu \mathrm{m} / \mathrm{s})$, VSL $(\mu \mathrm{m} / \mathrm{s})$, VCL $(\mu \mathrm{m} / \mathrm{s})$, and ALH $(\mu \mathrm{m})$ were significantly $(P<0.01)$ the best by using INRA 96 extender at rates of dilution of $1: 1(155.53 \pm 4.45,108.81 \pm 3.84,302.32$ \pm 6.96 , and $14.26 \pm 0.28$, respectively) and $1: 2$ (161.95 \pm 4.73 , $118.23 \pm 4.81,316.44 \pm 8.15$, and $14.89 \pm 0.32$, respectively) in comparison with the other two extenders used (TRIS -egg yolk and E-Z Mixin; Table 3). Table 4 shows that the percentage of stallions' sperm's progressive motility after thawing at $37^{\circ} \mathrm{C}$ for

Table 1: Semen Evaluation of the Arabian horses (mean \pm SEM).

\begin{tabular}{|l|l|}
\hline Semen parameters & Values \\
\hline Ejaculate volume (mL) & $60.00 \pm 3.34$ \\
\hline Overall motility (\%) & $88.54 \pm 2.42$ \\
\hline Progressive motility (\%) & $54.79 \pm 2.32$ \\
\hline Sperm cell concentration (x106/ mL) & $178.63 \pm 19.47$ \\
\hline Live sperm (\%) & $69.75 \pm 1.64$ \\
\hline Sperm cell abnormalities (\%) & $23.46 \pm 1.46$ \\
\hline Number of ejaculates $=48$ & \\
\hline
\end{tabular}

$30 \mathrm{sec}$ was significantly $(P<0.01)$ the best by using INRA Freeze extender at a rate of dilution of 1:2 (28.10 \pm 2.00$)$. The viability indexes of sperm's progressive motility after thawing at $37^{\circ} \mathrm{C}$ for $30 \mathrm{sec}$ are significantly higher $(P<0.01)$ for the INRA Freeze extender at dilution rates of $1: 1$ and $1: 2$ (50.92 \pm 5.71 and 68.83 \pm 6.77 , respectively) than for the TRIS -egg yolk and E-Z Mixin extenders (Table 4). The stallions' sperm motility characteristics after thawing at $37^{\circ} \mathrm{C}$ for 30 sec such as VAP $(\mu \mathrm{m} / \mathrm{s})$, VSL $(\mu \mathrm{m} / \mathrm{s})$, and VCL $(\mu \mathrm{m} / \mathrm{s})$ are the highest $(P<0.01)$ by using INRA Freeze extender at a rate of dilution $1: 2(113.19 \pm 3.49,89.87 \pm 3.47$, and $214.62 \pm 5.36$, respectively) when compared with INRA Freeze extender at a dilution rate of 1:1, TRIS -egg yolk and E-Z Mixin extenders (Table 5).

As shown in Table 6, the percentages of stallions' sperm's progressive motility after thawing at $75^{\circ} \mathrm{C}$ for $7 \mathrm{sec}$ are significantly $(P<0.01)$ higher for INRA Freeze and E-Z Mixin extenders at rates of dilution of $1: 1$ and 1:2 (23.55 \pm 1.92 and $21.09 \pm 1.79$, respectively for INRA Freeze, $19.40 \pm 1.07$ and 20.17 \pm 0.84 , respectively for E-Z Mixin extenders) than in the TRIS -egg yolk extender. The characteristics of sperm motility after thawing at $75^{\circ} \mathrm{C}$ for $7 \mathrm{sec}$ such as VAP $(\mu \mathrm{m} / \mathrm{s})$ and VSL $(\mu \mathrm{m} / \mathrm{s})$ are significantly $(P<0.01)$ the fastest by using INRA Freeze extender at a rate of dilution of 1:2 (107.06 \pm 3.7189 and $83.96 \pm 3.47$, respectively) in comparison with INRA Freeze at a dilution rate of 1:1, TRIS -egg yolk and E-Z Mixin extenders (Table 7). Table 8 shows the fertilizing capacity of preserved Arabian horse semen in the INRA Freeze extender and thawed at $37^{\circ} \mathrm{C}$ for $30 \mathrm{sec}$. The conception rate is significantly higher $(P<0.01)$ by using INRA Freeze extender at a dilution rate of $1: 1(50 \%)$ than it is at $1: 2$ (20\%), with total conception of $35 \%$ (Table 8). The effect of a number of inseminating doses on the fertilizing capacity of the cryopreserved Arabian horse semen in INRA Freeze extender and thawing at $37^{\circ} \mathrm{C}$ for $30 \mathrm{sec}$ is shown in Table 9. The use of two inseminating doses gives significantly $(P<0.01)$ better results $(50 \%)$ than does the use of one inseminating dose $(28.57 \%$; Table 9).

\section{Discussion}

The ejaculate volume of Arabian horse fresh semen was $60.00 \pm 3.34 \mathrm{ml}$. Similar values of ejaculate volumes ranging from 45 to $70 \mathrm{ml}$ were recorded by several authors in Arabians [26,27], and in thoroughbred and quarter horses [28,29]. The percentage of progressive motility in Arabian horses was 54.79 $\pm 2.32 \%$, which is lower than the percentage $(\leq 60)$ found in Arabian [26,30] and thoroughbred stallions [31,32]. Sperm cell concentration of Arabians was $178.63 \pm 19.47 \times 106 / \mathrm{ml}$. This is compared favorably with the reported range of $100-350 \mathrm{x}$ $106 / \mathrm{ml}$ [32]. Higher values have been recorded in Arab stallions ranging from 293.84 to $499.50 \times 106 / \mathrm{ml}[26,30,33]$ and a low value of $151.00 \times 106 / \mathrm{ml}$ has been found in Arabians [27]. In thoroughbred stallions, average sperm cell concentration ranged from 110.00 to 164.13 [34,35], from 200.00 to $282.00 \times 106 / \mathrm{ml}$ [36,37], and from 348.00 to $388.00 \times 106 / \mathrm{ml}$ [38]. In this study, the percentage of sperm cell abnormality was $23.46 \pm 1.46$. In Arabians, sperm cell abnormalities ranged from 30.40 to $44.70 \%$ $[26,30,33]$, whereas the percentage was 18.2 in thoroughbred stallions [39]. 
Table 2: Comparison of stallions' semen parameters (mean \pm SEM) in the different extenders at $5^{\circ} \mathrm{C}$.

\begin{tabular}{|l|l|l|l|l|l|}
\hline Extenders & Overall motility (\%) & Progressive motility (\%) & Concentration(x10 $/ \mathrm{mL})$ & Live sperm (\%) & Sperm abnormalities (\%) \\
\hline INRA 96 1:1 & $94.08^{\mathrm{a}} \pm 1.30$ & $39.54^{\mathrm{a}} \pm 2.50$ & $57.00^{\mathrm{a}} \pm 6.50$ & $63.68^{\mathrm{a}} \pm 1.54$ & $18.42^{\mathrm{a}} \pm 2.26$ \\
\hline INRA 96 1:2 & $91.71^{\mathrm{a}} \pm 0.95$ & $44.13^{\mathrm{a}} \pm 3.07$ & $49.00^{\mathrm{a}} \pm 5.59$ & $67.22^{\mathrm{a}} \pm 1.36$ & $27.33^{\mathrm{b}} \pm 1.18$ \\
\hline Tris-egg yolk 1:1 & $85.13^{\mathrm{bf}} \pm 2.87$ & $19.00^{\mathrm{b}} \pm 0.87$ & $81.76^{\mathrm{b}} \pm 4.90$ & $45.32^{\mathrm{b}} \pm 1.16$ & $23.21^{\mathrm{c}} \pm 0.42$ \\
\hline Tris-egg yolk 1:2 & $76.00^{\mathrm{de}} \pm 2.72$ & $16.04^{\mathrm{c}} \pm 0.80$ & $81.77^{\mathrm{b}} \pm 5.91$ & $41.18^{\mathrm{b}} \pm 0.82$ & $21.08^{\mathrm{d}} \pm 0.26$ \\
\hline E-Z mixin 1:1 & $85.96^{\mathrm{bd}} \pm 1.16$ & $33.83^{\mathrm{d}} \pm 1.16$ & $51.97^{\mathrm{a}} \pm 2.51$ & $67.40^{\mathrm{a}} \pm 1.55$ & $25.35^{\mathrm{bce}} \pm 1.08$ \\
\hline E-Z mixin 1:2 & $78.88^{\mathrm{ef}} \pm 1.90$ & $31.00^{\mathrm{d}} \pm 1.53$ & $79.04^{\mathrm{b}} \pm 5.92$ & $44.56^{\mathrm{b}} \pm 2.28$ & $24.60^{\mathrm{e}} \pm 0.42$ \\
\hline \multicolumn{2}{|l|}{ Means with dissimilar superscripts in the same column are significantly different at $P<0.01$} & &
\end{tabular}

Number of ejaculates $=48$

Table 3: Comparison of characters of sperm motility (mean \pm SEM) in the different extenders at $5^{\circ} \mathrm{C}$.

\begin{tabular}{|c|c|c|c|c|c|c|c|}
\hline Extenders & $\begin{array}{l}\text { Path velocity (VAP) } \\
\mu \mathrm{m} / \mathrm{s}\end{array}$ & $\begin{array}{l}\text { Progressive velocity } \\
\text { (VSL) } \mu \mathrm{m} / \mathrm{s}\end{array}$ & $\begin{array}{l}\text { Track speed } \\
\text { (VCL) } \mu \mathrm{m} / \mathrm{s}\end{array}$ & $\begin{array}{l}\text { Lateral amplitude } \\
\text { head (ALH) } \mu \mathrm{m}\end{array}$ & $\begin{array}{l}\text { Beat cross } \\
\text { frequency (BCF) Hz }\end{array}$ & $\begin{array}{l}\text { Straightness } \\
\text { (STR) } \\
(\%)\end{array}$ & $\begin{array}{l}\text { Linearity (LIN) } \\
(\%)\end{array}$ \\
\hline INRA 96 1:1 & $155.53^{\mathrm{a}} \pm 4.45$ & $\begin{array}{l}108.81^{\mathrm{a}} \\
\pm 3.84\end{array}$ & $\begin{array}{l}302.32^{\mathrm{a}} \\
\pm 6.96\end{array}$ & $\begin{array}{l}14.26^{\mathrm{a}} \\
\pm 0.28\end{array}$ & $\begin{array}{l}25.85^{\mathrm{a}} \\
\pm 0.71\end{array}$ & $\begin{array}{l}66.29^{\mathrm{ab}} \\
\pm 1.29\end{array}$ & $\begin{array}{l}34.48^{\mathrm{ab}} \\
\pm 0.67\end{array}$ \\
\hline INRA 96 1:2 & $161.95^{\mathrm{a}} \pm 4.73$ & $\begin{array}{l}118.23^{\mathrm{a}} \\
\pm 4.81\end{array}$ & $\begin{array}{l}316.44^{\mathrm{a}} \\
\pm 8.15\end{array}$ & $\begin{array}{l}14.89^{\mathrm{a}} \\
\pm 0.32\end{array}$ & $\begin{array}{l}27.36^{\mathrm{a}} \\
\pm 0.81\end{array}$ & $\begin{array}{l}69.53^{\mathrm{b}} \\
\pm 1.50\end{array}$ & $\begin{array}{l}36.31^{\mathrm{b}} \\
\pm 1.04\end{array}$ \\
\hline \begin{tabular}{|l|} 
Tris-egg yolk \\
1:1
\end{tabular} & $103.04^{b} \pm 3.97$ & $\begin{array}{l}64.70^{\mathrm{b}} \\
\pm 1.94\end{array}$ & $\begin{array}{l}197.10^{\mathrm{b}} \\
\pm 6.65\end{array}$ & $\begin{array}{l}10.90^{\mathrm{b}} \\
\pm 0.19\end{array}$ & $\begin{array}{l}27.30^{\mathrm{a}} \\
\pm 0.69\end{array}$ & $\begin{array}{l}58.64^{c} \\
\pm 0.80\end{array}$ & $\begin{array}{l}31.14^{c} \\
\pm 0.27\end{array}$ \\
\hline \begin{tabular}{|l|} 
Tris-egg yolk \\
$1: 2$
\end{tabular} & $88.44^{c} \pm 2.54$ & $\begin{array}{l}57.07^{c} \\
\pm 1.46\end{array}$ & $\begin{array}{l}177.48^{\mathrm{c}} \\
\pm 4.90\end{array}$ & $\begin{array}{l}11.09^{\mathrm{b}} \\
\pm 0.17\end{array}$ & $\begin{array}{l}29.58^{\mathrm{b}} \\
\pm 0.68\end{array}$ & $\begin{array}{l}59.80^{d} \\
\pm 0.61\end{array}$ & $\begin{array}{l}30.47^{d} \\
\pm 0.27\end{array}$ \\
\hline E-Z mixin 1:1 & $127.31^{\mathrm{d}} \pm 2.15$ & $\begin{array}{l}92.13^{\mathrm{d}} \\
\pm 2.19\end{array}$ & $\begin{array}{l}251.93^{\mathrm{d}} \\
\pm 3.13\end{array}$ & $\begin{array}{l}12.84^{c} \\
\pm 0.07\end{array}$ & $\begin{array}{l}34.13^{c} \\
\pm 0.28\end{array}$ & $\begin{array}{l}65.75^{\mathrm{a}} \\
\pm 0.60\end{array}$ & $\begin{array}{l}33.25^{\text {ae }} \\
\pm 0.45\end{array}$ \\
\hline E-Z mixin 1:2 & $128.69^{d} \pm 3.60$ & $\begin{array}{l}92.74^{\mathrm{d}} \\
\pm 3.32\end{array}$ & $\begin{array}{l}254.96^{d} \\
\pm 6.19\end{array}$ & $\begin{array}{l}12.81^{\mathrm{c}} \\
\pm 0.12\end{array}$ & $\begin{array}{l}33.17^{\mathrm{d}} \\
\pm 0.28\end{array}$ & $\begin{array}{l}65.00^{\mathrm{a}} \\
\pm 0.80\end{array}$ & $\begin{array}{l}33.14^{\text {ae }} \\
\pm 0.54\end{array}$ \\
\hline
\end{tabular}

Table 4: Comparison of stallions' semen parameters (mean \pm SEM) in the different extenders after thawing at $37^{\circ} \mathrm{C}$ for 30 sec and incubation at $30^{\circ} \mathrm{C}$.

\begin{tabular}{|c|c|c|c|c|c|c|c|}
\hline Extenders & $\begin{array}{l}\text { Overall } \\
\text { motility (\%) }\end{array}$ & $\begin{array}{l}\text { Progressive } \\
\text { motility (\%) }\end{array}$ & $\begin{array}{l}\text { Concentration } \\
\left(\times 10^{6} / \mathrm{mL}\right)\end{array}$ & $\begin{array}{l}\text { Live sperm } \\
\text { (\%) }\end{array}$ & $\begin{array}{l}\text { Sperm } \\
\text { abnormalities } \\
\text { (\%) }\end{array}$ & $\begin{array}{l}\text { Viability Index } \\
\text { Overall motility }\end{array}$ & $\begin{array}{l}\text { Viability Index } \\
\text { Progressive motility }\end{array}$ \\
\hline INRA Freeze 1:1 & $64.67^{\mathrm{a}} \pm 3.27$ & $22.75^{\text {acd }} \pm 2.36$ & $58.77^{a} \pm 3.63$ & $30.86^{\mathrm{a}} \pm 1.82$ & $21.57^{\mathrm{a}} \pm 1.07$ & $135.42^{\mathrm{a}} \pm 14.06$ & $50.92^{a} \pm 5.71$ \\
\hline INRA Freeze 1:2 & $60.00^{\mathrm{ab}} \pm 3.38$ & $28.10^{\mathrm{a}} \pm 2.00$ & $52.04^{a} \pm 3.59$ & $29.43^{\mathrm{a}} \pm 1.03$ & $31.43^{b} \pm 1.26$ & $147.42^{b} \pm 15.78$ & $68.83^{b} \pm 6.77$ \\
\hline Tris-egg yolk 1:1 & $60.80^{\text {ad }} \pm 3.29$ & $13.00^{\mathrm{b}} \pm 0.66$ & $74.10^{\mathrm{b}} \pm 2.55$ & $22.00^{\mathrm{b}} \pm 0.23$ & $27.00^{c} \pm 0.34$ & $97.67^{\mathrm{ce}} \pm 14.79$ & $17.58^{c} \pm 3.04$ \\
\hline Tris-egg yolk 1:2 & $47.10^{c} \pm 2.39$ & $12.40^{\mathrm{b}} \pm 0.51$ & $83.03^{c} \pm 3.23$ & $22.50^{\mathrm{b}} \pm 1.11$ & $25.50^{\mathrm{d}} \pm 0.34$ & $72.83^{\mathrm{d}} \pm 15.70$ & $12.25^{\mathrm{d}} \pm 2.99$ \\
\hline E-Z mixin 1:1 & $55.00^{\text {bd }} \pm 1.97$ & $17.83^{c} \pm 0.76$ & $70.93^{b} \pm 3.42$ & $27.60^{\mathrm{a}} \pm 0.95$ & $28.00^{\text {bce }} \pm 0.92$ & $106.25^{c} \pm 10.83$ & $47.75^{\mathrm{e}} \pm 4.89$ \\
\hline E-Z mixin 1:2 & $53.00^{\mathrm{bcd}} \pm 2.47$ & $22.67^{d} \pm 1.10$ & $62.88^{\mathrm{a}} \pm 3.63$ & $21.00^{\mathrm{b}} \pm 1.05$ & $27.80^{\mathrm{e}} \pm 0.36$ & $94.25^{\mathrm{e}} \pm 13.11$ & $36.16^{\mathrm{f}} \pm 3.90$ \\
\hline
\end{tabular}

Table 5: Comparison of characters of sperm motility (mean \pm SEM) in the different extenders after thawing at $37^{\circ} \mathrm{C}$ for 30 sec.

\begin{tabular}{|l|l|l|l|l|l|l|l|}
\hline Extenders & $\begin{array}{l}\text { Path velocity } \\
\text { (VAP) } \boldsymbol{\mu m} / \mathbf{s}\end{array}$ & $\begin{array}{l}\text { Progressive } \\
\text { velocity (VSL) } \\
\boldsymbol{\mu m} / \mathbf{s}\end{array}$ & $\begin{array}{l}\text { Track speed (VCL) } \\
\boldsymbol{\mu m} / \mathbf{s}\end{array}$ & $\begin{array}{l}\text { Lateral amplitude } \\
\text { head (ALH) } \boldsymbol{\mu m}\end{array}$ & $\begin{array}{l}\text { Beat cross frequency } \\
(\mathbf{B C F}) \\
\text { Hz }\end{array}$ & $\begin{array}{l}\text { Straightness } \\
\text { (STR) } \\
(\%)\end{array}$ & $\begin{array}{l}\text { Linearity (LIN) } \\
(\%)\end{array}$ \\
\hline INRA Freeze 1:1 & $94.85^{\mathrm{ad}} \pm 4.34$ & $71.15^{\mathrm{ae}} \pm 3.96$ & $184.54^{\mathrm{ae}} \pm 6.77$ & $11.25^{\mathrm{ad}} \pm 0.17$ & $34.21^{\mathrm{a}} \pm 0.39$ & $67.18^{\mathrm{a}} \pm 0.87$ & $35.36^{\mathrm{ad}} \pm 0.82$ \\
\hline INRA Freeze 1:2 & $113.19^{\mathrm{b}} \pm 3.49$ & $89.87^{\mathrm{b}} \pm 3.47$ & $214.62^{\mathrm{b}} \pm 5.36$ & $11.57^{\mathrm{a}} \pm 0.17$ & $34.12^{\mathrm{a}} \pm 0.38$ & $71.22^{\mathrm{c}} \pm 0.91$ & $38.33^{\mathrm{b}} \pm 0.79$ \\
\hline Tris-egg yolk 1:1 & $63.20^{\mathrm{c}} \pm 1.61$ & $43.17^{\mathrm{c}} \pm 1.03$ & $125.46^{\mathrm{c}} \pm 3.50$ & $9.7^{\mathrm{b}} \pm 0.17$ & $29.78^{\mathrm{b}} \pm 0.24$ & $66.56^{\mathrm{ab}} \pm 1.16$ & $37.00^{\mathrm{ab}} \pm 1.19$ \\
\hline Tris-egg yolk 1:2 & $65.07^{\mathrm{c}} \pm 1.19$ & $45.64^{\mathrm{d}} \pm 0.88$ & $133.94^{\mathrm{d}} \pm 2.72$ & $9.47^{\mathrm{b}} \pm 0.18$ & $31.58^{\mathrm{c}} \pm 0.24$ & $65.89^{\mathrm{ad}} \pm 0.46$ & $34.11^{\mathrm{d}} \pm 0.64$ \\
\hline E-Z mixin 1:1 & $83.48^{\mathrm{d}} \pm 1.86$ & $61.47^{\mathrm{e}} \pm 1.79$ & $170.12^{\mathrm{e}} \pm 2.95$ & $10.27^{\mathrm{c}}$ & $38.75^{\mathrm{d}} \pm 0.08$ & $64.50^{\mathrm{bd}} \pm 0.53$ \\
\pm 0.18 & $12.00^{\mathrm{c}} \pm 0.43$ \\
\hline E-Z mixin 1:2 & $89.18^{\mathrm{a}} \pm 2.57$ & $67.25^{\mathrm{a}} \pm 2.21$ & $179.10^{\mathrm{a}} \pm 7.80$ & $10.70^{\mathrm{d}} \pm 0.24$ & $36.08^{\mathrm{e}} \pm 0.23$ & $66.50^{\mathrm{a}} \pm 0.59$ & $33.67^{\mathrm{d}} \pm 0.34$ \\
\hline
\end{tabular}


Table 6: Comparison of stallions' semen parameters (mean \pm SEM) in the different extenders after thawing at $75^{\circ} \mathrm{C}$ for $7 \mathrm{sec}$ and incubation at $30^{\circ} \mathrm{C}$.

\begin{tabular}{|c|c|c|c|c|c|c|c|}
\hline Extenders & $\begin{array}{l}\text { Overall } \\
\text { motility (\%) }\end{array}$ & $\begin{array}{l}\text { Progressive } \\
\text { motility (\%) }\end{array}$ & $\begin{array}{l}\text { Concentration } \\
\left(\times 10^{6} / \mathrm{mL}\right)\end{array}$ & Live sperm (\%) & $\begin{array}{l}\text { Sperm } \\
\text { abnormalities } \\
\text { (\%) }\end{array}$ & $\begin{array}{l}\text { Viability Index } \\
\text { Overall motility }\end{array}$ & $\begin{array}{l}\text { Viability Index } \\
\text { Progressive } \\
\text { motility }\end{array}$ \\
\hline INRA Freeze 1:1 & $62.00^{\mathrm{a}} \pm 3.94$ & $23.55^{\mathrm{a}} \pm 1.92$ & $64.83^{\mathrm{abc}} \pm 6.05$ & $16.43^{\mathrm{ab}} \pm 1.25$ & $28.00^{\mathrm{ad}} \pm 0.88$ & $142.17^{a} \pm 9.54$ & $67.25^{a} \pm 3.39$ \\
\hline INRA Freeze 1:2 & $49.36^{\mathrm{cd}} \pm 3.14$ & $21.09^{b} \pm 1.79$ & $70.46^{a} \pm 5.32$ & $15.33^{b} \pm 0.66$ & $35.17^{\mathrm{b}} \pm 1.59$ & $102.92^{\mathrm{b}} \pm 4.33$ & $49.75^{b} \pm 2.21$ \\
\hline Tris-egg yolk 1:1 & $62.88^{a} \pm 4.77$ & $13.00^{c} \pm 0.65$ & $69.70^{\mathrm{ab}} \pm 2.55$ & $28.00^{c} \pm 0.67$ & $25.50^{c} \pm 0.38$ & $107.92^{\text {bd }} \pm 3.41$ & $19.42^{\mathrm{c}} \pm 1.78$ \\
\hline Tris-egg yolk 1:2 & $47.38^{c} \pm 3.46$ & $10.50^{\mathrm{d}} \pm 0.45$ & $70.00^{\mathrm{ab}} \pm 2.83$ & $17.25^{\mathrm{a}} \pm 0.61$ & $26.75^{\mathrm{d}} \pm 0.70$ & $84.33^{c} \pm 8.86$ & $14.83^{\mathrm{d}} \pm 0.98$ \\
\hline E-Z mixin 1:1 & $57.08^{\text {ad }} \pm 5.77$ & $19.40^{\mathrm{a}} \pm 1.07$ & $55.78^{c} \pm 1.52$ & $24.60^{\mathrm{d}} \pm 0.78$ & $21.20^{\mathrm{e}} \pm 0.53$ & $92.50^{c} \pm 7.26$ & $41.75^{e} \pm 3.43$ \\
\hline E-Z mixin 1:2 & $47.50^{\mathrm{bc}} \pm 3.38$ & $20.17^{\mathrm{a}} \pm 0.84$ & $71.39^{\mathrm{ab}} \pm 4.02$ & $18.60^{\mathrm{a}} \pm 0.97$ & $28.40^{\mathrm{a}} \pm 0.64$ & $113.75^{d} \pm 3.58$ & $41.50^{\mathrm{e}} \pm 3.96$ \\
\hline
\end{tabular}

Means with dissimilar superscripts in the same column are significantly different at $P<0.01$

Number of ejaculates $=48$

Table 7: Comparison of characters of sperm motility (mean \pm SEM) in the different extenders after thawing at $75^{\circ} \mathrm{C}$ for 7 sec.

\begin{tabular}{|c|c|c|c|c|c|c|c|}
\hline Extenders & $\begin{array}{l}\text { Path velocity } \\
\text { (VAP) } \mu \mathrm{m} / \mathrm{s}\end{array}$ & $\begin{array}{l}\text { Progressive } \\
\text { velocity (VSL) } \\
\mu \mathrm{m} / \mathrm{s}\end{array}$ & $\begin{array}{l}\text { Track speed } \\
\text { (VCL) } \mu \mathrm{m} / \mathrm{s}\end{array}$ & $\begin{array}{l}\text { Lateral } \\
\text { amplitude head } \\
\text { (ALH) } \mu \mathrm{m}\end{array}$ & $\begin{array}{l}\text { Beat cross } \\
\text { frequency (BCF) } \\
\mathrm{Hz}\end{array}$ & $\begin{array}{l}\text { Straightness } \\
\text { (STR) } \\
\text { (\%) }\end{array}$ & $\begin{array}{l}\text { Linearity (LIN) } \\
(\%)\end{array}$ \\
\hline INRA Freeze 1:1 & $100.40^{\mathrm{a}} \pm 2.97$ & $74.54^{a} \pm 2.86$ & $194.62^{\text {ad }} \pm 10.01$ & $11.20^{\mathrm{a}} \pm 0.16$ & $33.26^{\mathrm{a}} \pm 0.29$ & $66.20^{\mathrm{a}} \pm 0.86$ & $34.70^{\mathrm{a}} \pm 0.61$ \\
\hline INRA Freeze 1:2 & $107.06^{c} \pm 3.71$ & $83.96^{c} \pm 3.47$ & $208.89^{\mathrm{b}} \pm 11.09$ & $11.82^{\mathrm{b}} \pm 0.19$ & $34.83^{\text {be }} \pm 0.52$ & $69.80^{c} \pm 0.92$ & $35.70^{\mathrm{b}} \pm 0.64$ \\
\hline Tris-egg yolk 1:1 & $69.17^{\mathrm{b}} \pm 1.44$ & $46.39^{b} \pm 0.71$ & $140.51^{c} \pm 7.91$ & $9.76^{c} \pm 0.08$ & $30.69^{c} \pm 0.24$ & $64.43^{\mathrm{bd}} \pm 0.74$ & $33.29^{\mathrm{a}} \pm 0.60$ \\
\hline Tris-egg yolk 1:2 & $68.79^{b} \pm 1.57$ & $48.23^{b} \pm 1.37$ & $141.20^{c} \pm 8.40$ & $10.01^{\mathrm{d}} \pm 0.08$ & $30.64^{c} \pm 0.21$ & $67.14^{\mathrm{a}} \pm 0.92$ & $35.43^{\mathrm{ab}} \pm 1.02$ \\
\hline E-Z mixin 1:1 & $95.94^{\mathrm{ad}} \pm 1.16$ & $71.74^{\mathrm{a}} \pm 0.96$ & $194.96^{\mathrm{ab}} \pm 6.76$ & $12.88^{e} \pm 0.07$ & $38.04^{\mathrm{d}} \pm 0.14$ & $64.00^{\mathrm{d}} \pm 0.35$ & $31.60^{c} \pm 0.28$ \\
\hline E-Z mixin 1:2 & $92.07^{\mathrm{d}} \pm 2.65$ & $69.42^{\mathrm{a}} \pm 2.55$ & $183.83^{d} \pm 9.56$ & $11.40^{\mathrm{ab}} \pm 0.26$ & $35.95^{\mathrm{e}} \pm 0.19$ & $66.16^{\mathrm{ab}} \pm 0.65$ & $33.50^{\mathrm{a}} \pm 0.35$ \\
\hline
\end{tabular}

Table 8: Fertilizing capacity of the cryopreserved Arabian horse semen (Conception rate).

\begin{tabular}{|l|l|l|l|}
\hline $\begin{array}{l}\text { Dilution rate } \\
\text { with INRA 96 }\end{array}$ & $\begin{array}{l}\text { Number of } \\
\text { inseminated } \\
\text { mares }\end{array}$ & $\begin{array}{l}\text { Number of } \\
\text { conceived } \\
\text { mares }\end{array}$ & Conception rate \\
\hline $\mathbf{1 : 1}$ & 20 & 10 & $50 \%^{\mathrm{a}}$ \\
\hline $\mathbf{1 : 2 V}$ & 20 & 4 & $20 \%^{\mathrm{b}}$ \\
\hline Total & 40 & 14 & $35 \%^{\mathrm{c}}$ \\
\hline
\end{tabular}

Percentages with dissimilar superscripts in the same column are significantly different at $P<0.01$

Table 9: Fertilizing capacity of the cryopreserved Arabian horse semen (Effect of number of inseminating doses).

\begin{tabular}{|l|l|l|l|}
\hline $\begin{array}{l}\text { Dilution rate with } \\
\text { INRA 96 } \\
\text { (1:1+1:2) }\end{array} \quad \begin{array}{l}\text { Number of } \\
\text { inseminated } \\
\text { mares }\end{array}$ & $\begin{array}{l}\text { Number of } \\
\text { conceived } \\
\text { mares }\end{array}$ & $\begin{array}{l}\text { Conception } \\
\text { rate }\end{array}$ \\
\hline $\begin{array}{l}\text { One dose } \\
\text { insemination }\end{array}$ & 28 & 8 & $28.57 \%^{\mathrm{a}}$ \\
\hline $\begin{array}{l}\text { Two doses } \\
\text { insemination }\end{array}$ & $12 \mathrm{~V}$ & 6 & $50 \%^{\mathrm{b}}$ \\
\hline Total & 40 & 14 & $35 \%^{\mathrm{c}}$ \\
\hline
\end{tabular}

Percentages with dissimilar superscripts in the same column are significantly different at $P<0.01$

In the present study, the percentages of progressive motility, live sperm, and sperm cell abnormalities were better for the INRA 96 extender at rates of dilution of 1:1 and 1:2 than they were in the TRIS -egg yolk and E-Z Mixin extenders after cooling of stallions' semen to $5^{\circ} \mathrm{C}$. INRA 96 and E-Z Mixin are the extenders more routinely used to cool and ship stallion semen in Europe and the United States, respectively [40]. However, in dogs, the percentage of motile spermatozoa after 4 days' storage at $4^{\circ} \mathrm{C}$ is $44.2 \%$ and $24.5 \%$ for the TRIS - egg yolk (20\%) and INRA 96 extenders, respectively [41]. In boars, the percentage of motile spermatozoa, as assessed by CASA on diluted semen, offers detailed predictive information regarding litter size [40]. Stallions' sperm's progressive velocity VSL $(\mu \mathrm{m} / \mathrm{sec})$ at $5^{\circ} \mathrm{C}$ ranged from $57.07 \pm 1.46$ in the TRIS -egg yolk to $118.23 \pm 4.81$ in the INRA 96 extender. Stallion sperm velocity in Kenney's diluent at $38^{\circ} \mathrm{C}$ is $73.20 \pm 3.00 \mu \mathrm{m} / \mathrm{sec}$ [15].

In the current study, stallions' sperm's progressive motility $(28.10 \pm 2.00 \%)$ and viability indexes after thawing at $37^{\circ} \mathrm{C}$ for 30 sec were better by using INRA Freeze extender than for TRIS -egg yolk and E-Z Mixin extenders. INRA 96 extender is one of the best chemically defined media used for frozen semen processing in stallions $[4,42]$. However, the motilities of frozen -thawed semen processed in modified Tyrode's solution and mTALP medium as determined by CASA are $43.4 \%$ for Tori stallions and $42.3 \%$ for Estonian stallions [43]. In addition, TRIS was the best extender for cryopreservation of stallion semen [44]. TRIS -egg yolk based extenders provide higher total and progressive motilities, higher percentages of rapid sperm and intact membrane cells, and more efficiency with regard to preservation of bovine post thaw sperm viability and fertility [45]. Components of extenders 
that include egg yolk serve to prevent cold shock and provide membrane stabilization during the freeze-thawing process [46]. The stabilization of sperm membranes has an important role in improvement of post thawed sperm quality $[47,48]$. In boars, TRIX cell (IMV Technologies) is the best for long-term storage [49].

The stallions' sperm's progressive motility percentages after thawing at $75^{\circ} \mathrm{C}$ for $7 \mathrm{sec}$ were higher by using INRA Freeza and E-Z Mixin extenders than was the TRIS -egg yolk extender.

In the motility of stallion spermatozoa after cryopreservation, there is a highly significant $(P<0.0001)$ difference between INRA Freeza extender (48.9\%) and E-Z Mixin extender (38.6\%). The combination of INRA 96 and 4\% glycerol in a freezing medium gave the highest average post-thaw motility (51.5\%) [50]. Thawing of stallion semen preserved in extender INRA Freeza at $37^{\circ} \mathrm{C}$ for $30 \mathrm{sec}$ is more favorable. Thawing at $75^{\circ} \mathrm{C}$ for $7 \mathrm{sec}$ slightly increased motility after $120 \mathrm{~min}$ at $37^{\circ} \mathrm{C}$ but it had no effect on per-cycle fertility [8].

The stallions' sperm motility characteristics such as VAP $(\mu \mathrm{m} / \mathrm{s})$ and VSL $(\mu \mathrm{m} / \mathrm{s})$ after thawing at both $37^{\circ} \mathrm{C}$ for $30 \mathrm{sec}$ and $75^{\circ} \mathrm{C}$ for $7 \mathrm{sec}$ are significantly $(P<0.01)$ the highest by using INRA Freeza extender when compared with TRIS -egg yolk and E-Z Mixin extenders. On the contrary, no correlations have been detected between fertility and motility immediately after thawing. CASA has replaced light microscopy in the evaluation of frozen -thawed stallion sperm, but so far, no one has been able to demonstrate a correlation between the fertility of frozen stallion semen and any of the motility parameters obtained by those instruments [51].

In this study, the conception rate was higher by using INRA Freeza extender (thawed at $37^{\circ} \mathrm{C}$ for $30 \mathrm{sec}$ ) at a dilution rate of $1: 1(50 \%)$ than it was at a dilution rate of $1: 2(20 \%)$, with total conception of $35 \%$. In the same direction, INRA 96 extender supplemented with egg yolk and glycerol significantly improved per-cycle pregnancy rates in mares compared with INRA Freeza extender $(71 \%$ versus $40 \%, P<0.01)$ [52]. The conception rate of frozen -thawed stallion semen ranged from 49.4 to $53.5 \%$ [53]. The use of two inseminating doses of frozen stallion semen gave better results $(50 \%)$ than did the use of one inseminating dose $(28.57 \%)$. Nevertheless, no differences were observed in pregnancy rates for mares inseminated once or multiple times in a given cycle $(51.5 \%$ versus $51.7 \%$ for study one and $47.1 \%$ versus $46.1 \%$ for study two). Mares inseminated twice in a cycle, once before ovulation and once after ovulation, become pregnant at a rate similar to that of mares inseminated once within $6 \mathrm{~h}$ postovulation (48.1\% versus 47.3\%) [54]. Acceptable fertility with frozen stallion semen is possible with the use of good quality semen, with a specific selection of mares and stallions, and with good mare management $[53,55]$. In conclusion, the fertilizing capacity of cryopreserved Arabian horse semen was best by using INRA Freeza extender at a dilution rate of 1:1 and in two insemination doses.

\section{Conflicts of Interest}

The authors wish to confirm that there are no known conflicts of interest associated with this publication and there has been no significant financial support for this work that could have influenced its outcome.

The authors confirm that the manuscript has been read and approved by all named authors and that there are no other persons who satisfied the criteria for authorship but are not listed. We further confirm that the order of authors listed in the manuscript has been approved by all of us.

\section{References}

1. Stout EAT. Equine embryo transfer: review of developing potential. Equine Vet J. 2006;38:467-478.

2. Kraemer DC. A History of Equine Embryo Transfer and Related Technologies. J Equine Vet Sci. 2013;33:305-308.

3. Pycock JF. Artificial insemination. Proceedings of the $10^{\text {th }}$ International Congress of World Equine Veterinary Association, Russia: Moscow; 2008.

4. Barbacini S. Frozen semen processing and quality control. Proceedings of the $12^{\text {th }}$ International Congress of World Equine Veterinary Association WEVA, Hyderabad, India; 2011.

5. Samper JC, Vidament M, Katila T, Newcombe J, Estrada A, Sargeant J. Analysis of some factors associated with pregnancy rates of frozen semen: A multi-centre study. Theriogenology. 2002;58:647-50.

6. Alvarenga MA, Papa FO, Landim-Alvarenga FC, Medeiros ASL. Amides as cryoprotectants for freezing stallion semen. Anim Reprod Sci. 2005;89:105-13.

7. Vidament M, Dupere AM, Julienne P, Evain A, Noue P, Palmer E. Equine frozen semen: Freezability and fertility field results. Theriogenology. 1997;48:905-17.

8. Vidament M, Yvon JM, Couty I, Arnaud G, Nguekam-Feugang J, Noue $\mathrm{P}$, et al. Advances in cryopreservation of stallion semen in modified INRA82. Anim Reprod Sci. 2001;68:201-18.

9. Alvarenga MA, Le ao KM, Papa FO, Landim-Alvarenga FC, Medeiros ASL, Gomes GM. The use of alternative cryoprotectors for freezing stallion semen. In: Proceedings of Workshop on Transporting Gametes and Embryos, Havemeyer Foundation; 2003; Brewster, Massachusetts, USA; 2003.p. 74-6.

10.Samper JC, Morris CA. Current methods for stallion semen cryopreservation: A survey. Theriogenology. 1998;49:895-903.

11. Ranzenigo G, Schulman M, Luther I. Effects of three semen extenders on the motility of frozen-thawed stallion sperm. Veterinary Europian Equine Meeting, Venice, Italy: XIV SIVE CONG; 2008

12. Samper JC. Artificial insemination with fresh and cooled semen In: Samper JC, editor. Equine Breeding Management and Artificial Insemination. Saunders, Elsevier Inc, Missouri; 2009.p. 165-74.

13. Pojprasath T, Lohachit C, Techakumphu M, Stout T, Tharasanit T. Improved cryopreservability of stallion sperm using a sorbitol-based freezing extender. Theriogenology 2011;75:1742-9.

14. Colenbrander B, Gadella BM, Stout TAE. The predictive value of semen analysis in the evaluation of the stallion fertility. Reprod Domest Anim. 2003; 38:305-11.

15. Tejerina F, Morrell J, Petterson J, Dalin AM, Rodriguez-Martinez H. Routine assessment of motility of ejaculated stallion spermatozoa using a novel computer-assisted motility analyzer (Qualisperm ${ }^{\mathrm{TM}}$ ). Anim Reprod. 2009;6:380-85. 
16. Belling TH. Postovulation breeding and related reproductive phenomena in the mare. Equine Prect. 1984;6:12-9.

17. Woods J, Bergfelt DR, Ginther OJ. Effects of time of insemination relative to ovulation on pregnancy rate and embryonic-loss rate in mares. Equine Vet J. 1990;22:410-15.

18. Huhtinen M, Koskinen E, Skidmore JA, Allen WR. Recovery rate and quality of embryos from mares inseminated after ovulation Theriogenology. 1996;45:719-26.

19. Shepherd C. Production and use of frozen stallion semen in the UK. Proceedings of the $47^{\text {th }}$ British Equine Veterinary Association Congress BEVA, Liverpool, United Kingdom: 2008.

20. Frunza I, Cernescu H, Mircu C, Otava G, Zarcula S, Gabriela Korodi G. Determination of the motility parameters of boar semen with CASA Faculty of Veterinary Medicine Bucharest, Scientific Works C Series LII; 2007. p. 73-9.

21. Blanchard TL, Varner DD, Schumacher J, Love CC, Brinsko SP, Rigby SL. Examination of the stallion for breeding soundness In: Blanchard L, Varner DD, Schumacher J, Love CC, Brinsko SP, Rigby SL, editors. Manual of Equine Reproduction. $2^{\text {nd }}$ ed. MOSBY; Elsevier Inc; Missouri 2003. p. 142-63.

22. Mortimer ST. A critical review of the physiological importance and analysis of sperm movement in mammals. Hum Reprod Update. 1997;3:403-39.

23. Milovanov VK, Trubkin GD, Chubenko NS, Tsvetkov IV, Erzin ZK, Meschankin AB. Artificial insemination of livestock in the U.S.S.R Israel Program For Scientific Translations, Jerusalem; 1964. p. 102-4.

24. Sanchez R, Gomez I, Samper JC. Artificial insemination with frozen semen. In: Samper JC, editor. Equine Breeding Management and Artificial Insemination. $2^{\text {nd }}$ ed. Saunders; Elsevier Inc; Missouri: 2009. p. $175-83$.

25 SPSS Inc. Statistical package for social sciences, version 22.0, for Windows (Microsoft, Redmond, WA), Chicago, IL: 2013.

26 Hemeida NA, El-Baghdady YRM, El-Belely MS, Ismail ST, AbouAhmed MM. Seminal characteristics of Arab stallions. Proceedings of the $4^{\text {th }}$ Scientific Congress, Faculty of Veterinary Medicine, Assiut University: 1990. p. 477-87.

27. Ball BA, Mohammed HO. Morphometry of stallion spermatozoa by computer - assisted image analysis. Theriogenology. 1995;44:367-77.

28. Love CC, Varner DD, Thompson JA. Intra- and inter stallion variation in sperm morphology and their relationship with fertility. J Reprod Fertil Suppl. 2000;56:93-100.

29. Sieme H, Bonk A, Hamann H, Klug E, Katila T. Effects of different artificial insemination techniques and sperm doses on fertility of normal mares and mares with abnormal reproductive history. Theriogenology. 2004;62:915-28.

30. Waheed MM, Ghoneim IM, Abdou MSS. Morphometric Characteristics of Spermatozoa in the Arabian horse with regard to season, age, sperm concentration, and fertility. J Equine Vet Sci. 2015;35:244-49.

31. Gahne S, Gånheim A, Malmgren L. Effect of insemination dose on pregnancy rate in mares. Theriogenology. 1998;49:1071-74

32. Sieme H. Semen evaluation, In: Samper JC, editor. Equine Breeding Management and Artificial Insemination. $2^{\text {nd }}$ ed. Saunders, Elsevier Inc; Missouri: 2009. p. 57-73.

33.El-Baghdady YRM, Abou-Ahmed MM, El-Belely MS, Hemeida NA Ismail ST. Influence of season on seminal characteristics and sexual behavior in Arab horses. Proceedings of the $4^{\text {th }}$ Scientific Congress, Faculty of Veterinary Medicine, Assiut University: 1990. p. 488-98.

34. Oba E, Bicudo SD, Pimentel SL, Lopes RS, Simonetti F, Hunziker RA. Quantitative and qualitative evaluation of stallion semen. Revista Brasileira de Reproduçäo Anim. 1993;17:57-74.

35. Dowsett KF, Knott LM. The influence of age and breed on stallion semen. Theriogenology. 1996;46:397-412.

36. Pickett BW, Hopwood ML, Faulkner LC. Variation in stallion semen characteristics. J Anim Sci. 1971;33:264.

37. Pickett BW, Faulkner LC, Seidel GE Jr, Berndtson WE, Voss JL. Reproductive physiology of the stallion. VI. Seminal and behavioral characteristics. J Anim Sci. 1976;43:617-25.

38. Pickett BW. Seasonal variation of stallion semen. Proceedings of the 3rd Technology Conference of Artificial Insemination and Reproduction, Chicago, Ill., USA; 1970. p. 52-7.

39. Hendrikse J. The semen of stallions of normal fertility. Tijdschr Diergeneesk. 1966;91:300-13.

40. Pinheiro ACS, Pamplona JF, Papa FO, Alvarenga MA. Comparison between three extenders for cooling of stallion semen before freezing. Anim Reprod Sci. 2008;107:340-41.

41. Bencharif D, Amirat-Briand L, LeGuillou J, Vialtelle C, Anton M, Schmitt $\mathrm{E}$, et al. Refrigeration of canine sperm at $+4^{\circ} \mathrm{C}$ : Comparative study of four different extenders for the refrigeration of canine sperm at $+4^{\circ} \mathrm{C}$ : LDL, Tris egg yolk, Equex $\AA^{\circledR}$, and INRA96 ${ }^{\circledR}$. Revue Méd Vét. 2013; $164: 252-62$

42. Vyt P, Maes D, Quinten C, Rijsselaere T, Deley W, Aarts M, et al Detailed motility evaluation of boar semen and its predictive value for reproductive performance in sows. Vlaams Diergeneeskundig Tijdschrift. 2008;77:291-98.

43. Kavak A, Johannisson A, Lundeheim N, Rodriguez-Martinez H, Aidnik M, Einarsson S. Evaluation of cryopreserved stallion semen from Tori and Estonian breeds using CASA and flow cytometry. Anim Reprod Sci. 2003;76:205-16.

44. Samper JC. Evaluation of cryopreserved semen : An alternative assay. Acta Vet Scand Suppl. 1992;88:59-65.

45. Crespilho AM, Filho MFSa', Dell'Aqua Jr JA, Nichi M, Monteiro GA, Avanzi BR, et al. Comparison of in vitro and in vivo fertilizing potential of bovine semen frozen in egg yolk or new lecithin based extenders. Livestock Sci. 2012;149:1-6.

46. Emamverdi M, Zhandi M, Zare Shahneh A, Sharafi M, Akhlaghi A Khodaei Motlagh M, et al. Flow cytometric and microscopic evaluation of post-thawed ram semen cryopreserved in chemically defined home-made or commercial extenders. Anim Prod Sci. 2014;55:551-8.

47. Maxwell WMC, Watson PF. Recent progress in the preservation of ram semen. Anim Reprod Sci. 1996;42:55-65.

48. Barbas JP, Mascarenhas RD. Cryopreservation of domestic animal sperm cells. Cell and Tissue Banking. 2009;10:49-62.

49. Lange-Consiglio A, Meucci A, Cremonesi F. Fluorescent multiple staining and CASA system to assess boar sperm viability and membranes integrity in short and long-term extenders. Open Vet J. $2013 \cdot 3 \cdot 21-35$

50. Scherzer J, Fayrer-Hosken RA, Aceves M, Hurley DJ, Ray LE, Jones L, Heusner GL. Freezing equine semen: the effect of combinations of semen extenders and glycerol on post-thaw motility. Aust Vet J. 2009;87:275-9. 
51. Katila T. In Vitro Evaluation of Frozen-Thawed Stallion Semen: A Review. Acta Vet Scand. 2001;42:199-217.

52 Pillet E, Batellier F, Duchamp G, Furstoss V, Vern YL, Kerboeuf D, et al Freezing stallion semen in INRA $96^{\circledR}$-based extender improves fertility rates in comparison with INRA82. Dairy Sci Technol. 2008;88:257-65.

53. Loomis PR. The equine frozen semen industry. Anim Reprod Sci. 2001;68:191-200.
54. Loomis PR, Squires EL. Frozen semen management in equine breeding programs. Theriogenology. 2005;64:480-91.

55. Camargo CE, Weiss RR, Kozicki LE, Duarte MP, Duarte MCG, Lunelli $D$, et al. Some factors affecting the rate of pregnancy after embryo transfer derived from the Brazilian Jumper Horse Breed. J Equine Vet Sci. 2013;33:924-9. 